\title{
A PAIRING RESTRICTION AT PACHYTENE UPON MULTIVALENT FORMATION IN AUTOTETRAPLOIDS
}

\author{
J. N. TIMMIS and H. REES \\ Department of Agricultural Botany, University College of Wales, Aberystwyth
}

Received 28.v.70

\section{INTRODUGTION}

$I_{\mathrm{T}}$ is very well known that the distribution of the different kinds of chromosome configurations, multivalents, bivalents and univalents, at first metaphase of meiosis in autotetraploids depends upon the frequency and location of chiasmata between homologous chromosomes. Durrant (1960) has derived formulae which predict the frequencies of the various types of configurations with varying chiasma frequencies. One of the assumptions of the model upon which these theoretical predictions were based was that a random association, involving all four chromosomes of each homologous set, was obligatory at pachytene. The same assumption underlies a later model put forward by John and Henderson (1962).

The following evidence in rye shows that pachytene associations do not, in fact, invariably involve all chromosomes of homologous sets. In the plants investigated pachytene association is exclusively between " pairs " of chromosomes in about one out of the seven homologous sets in each pollen mother cell (p.m.c.). This restriction to "pairs" contributes to the characteristically high frequency of bivalents, at the expense of multivalents, in rye autotetraploids.

\section{Materials AND methods}

Tetraploids. The four autotetraploids used were those produced by Hazarika and Rees (1967). They are derived from four inbred, homozygous lines $P_{1}, P_{8}, P_{12}$ and $P_{13}$ (see Rees, 1955).

Trisomic. The data on trivalent formation come from a single plant which turned up in a " normal " cross-pollinating rye population.

Interchanges. The two, independent, interchanges scored have been described in detail by Thompson (1956) and Rees (1961). Interchange $A$ involves chromosomes which organise the nucleolus, interchange $\mathrm{B}$ does not. The break points in both are near to the centromeres.

Pollen mother cells (p.m.c.'s) were squashed and stained in aceto-carmine.

\section{REsults}

\section{A. Autotetraploids}

Chiasmata in rye are located distally. If we assume $(a)$ that the four homologous chromosomes of each set or more specifically, in view of the distal localisation of chiasmata, the ends of the four homologues associate at random at pachytene (fig. l $a$ and $b$ ), $(b)$ at least one chiasma forms between at least three of the four paired ends, the configurations at metaphase 


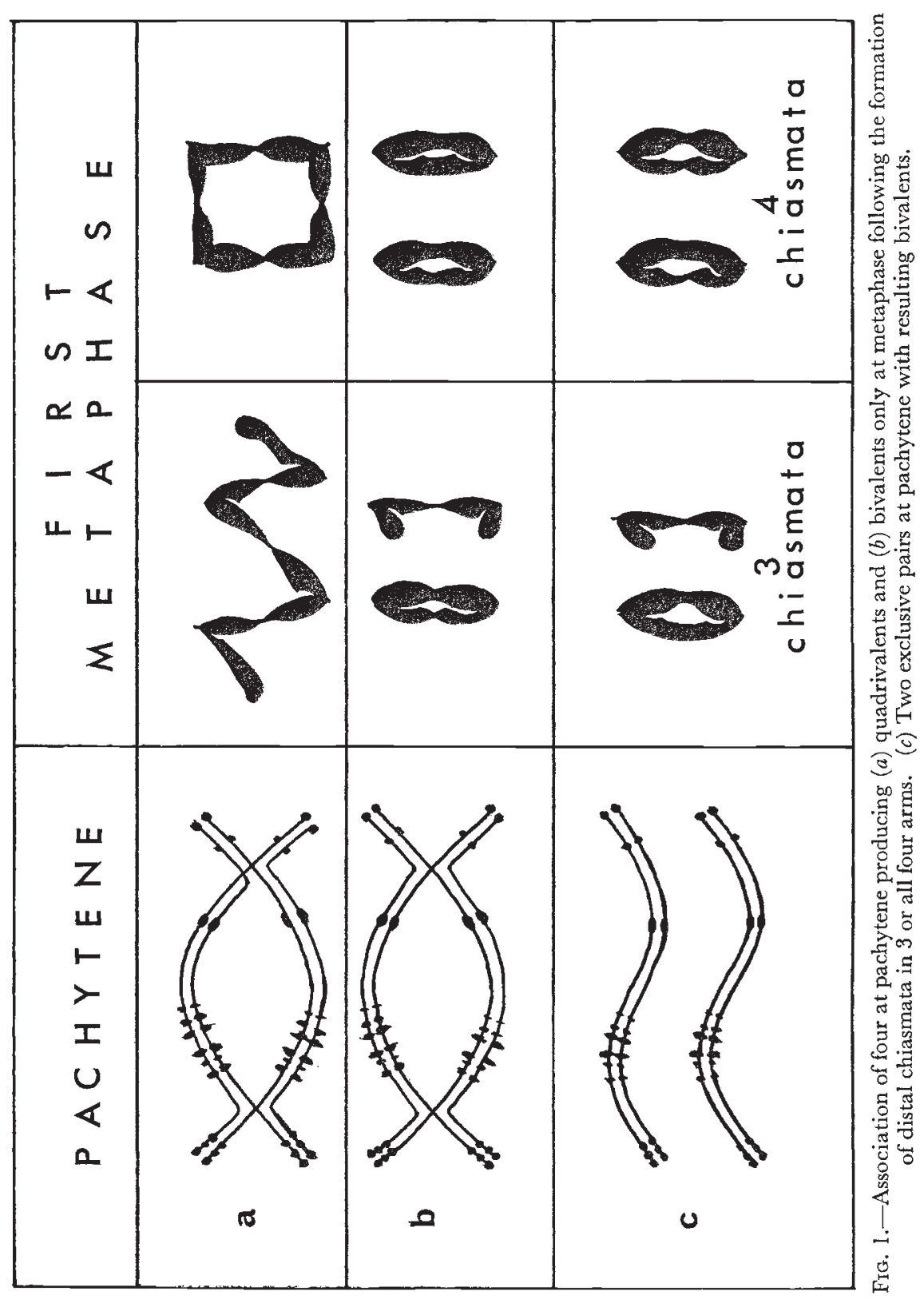


(table 1) will comprise quadrivalents and bivalents in the ratio of $1: 1$, with no trivalents (see John and Henderson, 1962).

In theory it would be an easy matter to find out to what extent $a$ is valid if we could be certain of distal chiasmata in at least three of four paired arms as required under $b$. In rye, however, this presents a practical difficulty because we cannot identify individual chromosomes at meiosis and, consequently, cannot readily determine the frequency and distribution of chiasmata for each set of four homologues. The difficulty is resolved by selecting,

TABLE 1

The distributions of quadrivalents (IVS) and bivalents (IIs) at first metaphase of meiosis in an autotetraploid assuming (a) pairing is initiated at the ends of the chromosomes at pachytene, (b) that pairing between ends is at random and (c) that at least one distal chiasma is formed in at least three of the four arms. The four homologous chromosomes are represented by $A^{1} B^{1}, A^{2} B^{2}, A^{3} B^{3}, A^{4} B^{4}$, where $A^{1}$ is homologous with $A^{2}, A^{3}$ and $A^{4}$ and $B^{1}$ with $B^{2}, B^{3}$ and $B^{4}$. After John and Henderson (1962)

Pachytene
associations
at B $\left\{\begin{array}{lccc}\mathrm{B}^{1} \mathrm{~B}^{2} / \mathrm{B}^{3} \mathrm{~B}^{4} & \mathrm{II}+\mathrm{II} & \mathrm{A}^{1} \mathrm{~A}^{3} / \mathrm{A}^{2} \mathrm{~A}^{4} & \mathrm{~A}^{1} \mathrm{~A}^{4} / \mathrm{A}^{2} \mathrm{~A}^{3} \\ \mathrm{~B}^{1} \mathrm{~B}^{3} / \mathrm{B}^{2} \mathrm{~B}^{4} & \text { IV } & \text { IV } & \text { II + II } \\ \mathrm{B}^{1} \mathrm{~B}^{4} / \mathrm{B}^{2} \mathrm{~B}^{3} & \text { IV } & \text { IV } & \text { IV } \\ & \text { IVs : IIs : : I : }: 1\end{array}\right.$

for metaphase scoring, p.m.c.'s, (a) which contain not more than one rod bivalent, the remaining bivalents being rings (plate-fig. $1 a),(b)$ in which any univalents present may be "allocated" to trivalents.

The high chiasma frequency of this p.m.c. sample guarantees that neither asynapsis or ineffective pairing at pachytene nor a subsequent deficiency of chiasmata was limiting to the formation of multivalents.

The distributions of multivalents and bivalents observed in such a selected sample of p.m.c.'s are given in table 2. (Over 94 per cent. of the multivalents

TABLE 2

The mean frequencies of multivalents and bivalents at first metaphase in a sample of p.m.c.'s with high chiasma frequencies in autotetraploids of rye. Data from at least 36 p.m.c.'s in each line

$\begin{array}{lcc}\text { Line } & \text { Multivalents } & \text { Bivalents } \\ \mathrm{P}_{1} & 4 \cdot 2 & 5 \cdot 6 \\ \mathrm{P}_{8} & 3 \cdot 9 & 6 \cdot 2 \\ \mathrm{P}_{12} & 4 \cdot 1 & 5 \cdot 8 \\ \mathrm{P}_{13} & 4 \cdot 1 & 5 \cdot 8 \\ \text { Means } & 4 \cdot 1 & 5 \cdot 8\end{array}$

are quadrivalents. The few trivalents which appear are assumed to arise from failure of chiasmata formation in one arm of a multiple association of four at pachytene.) Of particular relevance is that, in all four lines, bivalents outnumber multivalents. The "excess" of bivalents over the expected 50 per cent. (table 1$)$ is highly significant $\left(\chi_{1}^{2}=79 \cdot 87, \mathrm{P}=<0 \cdot 001\right)$. We conclude, therefore, that a random association of ends, involving all four chromosomes of each homologous set is not obligatory at pachytene. We conclude further that the pachytene associations are, to a significant degree, restricted to exclusive pairs (fig. $1 c$; cf. $\mathrm{l} a$ and $b$ ), a possibility recognised long ago by Darlington (1937) and others. There are no grounds to sustain 
the alternative explanation, namely that some "interference" promotes, to a disproportionate degree, the reassociation of the same pairs of chromosomes at each end, as in $1 b$.

The extent to which the chromosomes associate exclusively in "pairs" at pachytene is readily estimated. The number of bivalents derived from random associations of four at pachytene equals that of multivalents, namely $4 \cdot 1$ (table 2). The remainder, $1 \cdot 7$ of the $5 \cdot 8$ bivalents per cell (table 2), derive therefore from pachytene "pairs". This means that the chromosomes of one of the seven homologous sets in nearly every cell associate exclusively in " pairs" at pachytene.

\section{TABLE 3}

The distributions of chromosome configurations per cell at first metaphase of meiosis in a random sample of p.m.c.'s in four autotetraploid ryes. Data pooled from scoring 80 p.m.c.'s in each of two plants in each line

\begin{tabular}{|c|c|c|c|c|}
\hline \multirow[b]{2}{*}{ Line } & \multicolumn{2}{|c|}{ Multivalents } & \multicolumn{2}{|c|}{ Bivalents and univalents } \\
\hline & IV & III & II & $\overrightarrow{1}$ \\
\hline $\mathbf{P}_{1}$ & $3 \cdot 2$ & $0 \cdot 2$ & $7 \cdot 0$ & $0 \cdot 5$ \\
\hline$P_{8}$ & $3 \cdot 4$ & $0 \cdot 2$ & $6 \cdot 8$ & $0 \cdot 3$ \\
\hline$P_{12}^{0}$ & $3 \cdot 1$ & $0 \cdot 4$ & $6 \cdot 7$ & $0 \cdot 8$ \\
\hline $\mathbf{P}_{13}$ & $3 \cdot 3$ & $0 \cdot 2$ & $6 \cdot 7$ & $0 \cdot 6$ \\
\hline Means & & & & \\
\hline
\end{tabular}

The conclusion that chromosome association is occasionally exclusively restricted to "pairs" at pachytene is, of course, based on a highly selected sample in so far as the cells contained bivalents with high chiasma frequencies, rings rather than rods. It is important to emphasise, however, that there was no question of a bias towards cells with a high frequency of bivalents and it is the frequency which is relevant to this issue. The absence of bias towards a high bivalent frequency is confirmed by reference to the chromosome distributions in a random sample of p.m.c.'s (table 3). Here, in fact, the bivalent frequency is higher, that of multivalents lower, than in table 2. This difference is, of course, not surprising in view of the lower chiasma frequency in these cells.

Confirmation of the tendency among polysomic chromosome sets to form pairs at pachytene is provided by the following observations in trisomic rye.

\section{B. A Trisomic}

Assuming, (a) a random association between the arms of the three homologous chromosomes at pachytene, $(b)$ the formation of at least one distal chiasma in two separate arms, the relative frequencies of trivalents and of bivalents with univalents to be expected at metaphase from the three homologous chromosomes in a trisomic are given in table 4. They are 2:1 respectively. We present in table 5 the distributions of trivalents and bivalents in p.m.c.'s which satisfy the assumption under $b$, namely where at least one distal chiasma formed in two separate arms. Such cells are easily selected. They include all cells containing trivalents and cells in which all bivalents are rings (plate-figs. $1 b$ and $c$ ). The ratio of trivalents 
to bivalents is widely different from the expected $2: 1$. As in the autotetraploids there is, in fact, a highly significant excess of bivalents $\left(\chi_{1}^{2}=9 \cdot 34\right.$, $P=<0.01)$. Clearly association between the arms of homologous chromosomes at pachytene is not at random. There is a tendency to form a pair to the exclusion of the third.

\section{TABLE 4}

The distributions of trivalents (IIIs) and bivalents (IIs) with univalents (Is) at first metaphase of meiosis in trisomics assuming that (a) pairing at pachytene is initiated at the ends of the chromosomes, (b) that pairing between ends is at random and (c) that at least one distal chiasma is formed in each of the arms paired. The homologous chromosomes are represented by $A^{1} B^{1}, A^{2} B^{2}, A^{3} B^{3}$ where $A^{1}$ is homologous with $A^{2}$ and $A^{3}$ and $B^{1}$ with $B^{2}$ and $B^{3}$

Pachytene
associations
at $B$$\left\{\begin{array}{llll}\mathrm{A}^{1} \mathrm{~A}^{2} & \mathrm{~A}^{1} \mathrm{~A}^{3} & \mathrm{~A}^{2} \mathrm{~A}^{3} \\ \mathrm{~B}^{1} \mathrm{~B}^{3} & \text { II I } & \text { III } & \text { III } \\ \mathrm{B}^{2} \mathrm{~B}^{3} & \text { III } & \text { II }+ \text { I } & \text { III } \\ & \text { III } & \text { III } & \text { II }+ \text { I }\end{array}\right.$

The number of bivalents per cell derived from random associations of all three homologues (see table 4 ) will be half the number of trivalents, namely $0 \cdot 27$. The remainder, $0 \cdot 19$, represents directly the proportion of exclusive "pairs" at pachytene. It will be recalled that the estimate of the number of homologous sets forming " pairs" at pachytene in autotetraploids was nearly $1(0.85)$ out of 7 , a ratio of 0.12 . It would be rash to attach too much significance to the fact that the tendency to form "pairs" at

TABLE 5

The relative frequencies of trivalents and of ring bivalents with univalents at first metaphase of meiosis in trisomic rye

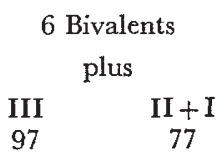

pachytene in the trisomic is even higher than in autotetraploids $(0.19$ as compared with $0 \cdot 12$ per homologous set) because data on the trisomic are, after all, derived from p.m.c.'s trisomic for one particular chromosome of the complement.

\section{Interchange heterozygotes}

Implicit in the data on autotetraploids is that a single association involving all four homologous chromosomes of a set is not obligatory even where pachytene pairing is complete. In an interchange heterozygote the situation for the multiple of four "interchange" chromosomes is altogether different. For complete pairing, association must inevitably involve all four chromosomes. Unless the chiasma frequency is limiting, that is less than one in each of three arms, a "quadrivalent" at metaphase is inevitable. Table 6 shows, in fact, the expected high frequency of "quadrivalents", 759 in a random sample of 800 p.m.c.'s (95 per cent.) in our rye interchanges. 
It is instructive, however, to consider p.m.c.'s in which the chiasma frequency is limiting, that is limited to one in each of two arms. Under these circumstances we would expect, following association of all four chromosomes at pachytene, "trivalents" (with univalents) to be twice as frequent as a pair of " bivalents" at first metaphase. Table 6 shows only 17 " trivalents" as compared with 24 pairs of "bivalents". The departure from a two to one ratio is highly significant $\left(\chi_{1}^{2}=11 \cdot 72, \mathrm{P}=<0 \cdot 001\right)$. To explain the deficit of "trivalents" we must conclude that even in interchange heterozygotes there is some restriction of pachytene associations to separate " pairs" of chromosomes, even though these are only partly homologous.

TABLE 6

The distributions of IVs, IIIs, with Is, and two IIs at first metaphase of meiosis in interchange heterozygotes in rye. Data pooled from 400 metaphases for each of two interchanges, $\mathrm{A}$ and $\mathrm{B}$

$\begin{array}{lcc} & 5 \text { Bivalents } \\ & \text { plus } & \\ \text { IV } & \text { III + I } & \text { II + II } \\ 759 & 17 & 24\end{array}$

From the mechanical standpoint it appears highly probable that once pairing is initiated between the two ends of homologous chromosomes the pairing tends to "zip" along the same pair, to the occasional exclusion of other homologous partners in interchange heterozygotes even at the cost of illegitimate or " torsion" pairing (Darlington, 1937).

\section{Discussion}

Under the special but by no means uncommon conditions which apply in rye, viz. the initiation of pachytene pairing at the ends of chromosomes and, subsequently, the formation of distal chiasmata, the model of John and Henderson (1962) is especially appropriate. It goes a very long way, but not quite all the way, towards explaining the apparent paucity of multivalents, particularly of trivalents, at meiosis in the autotetraploids, a paucity widely reported among other species (see Callan, 1949; Morrison and Rajhathy, 1960; John and Lewis, 1962). John and Henderson's model assumes, however, a random association within each homologous set at pachytene. In rye, as we have shown, there is an element of non-randomness in that some of the homologous chromosomes associate in exclusive pairs at pachytene, thereby adding to the quota of bivalents and accentuating further the "shortage" of multivalents at metaphase.

In order to account completely, or predict in detail, the distribution of multivalents, bivalents and univalents at metaphase of meiosis in autotetraploids we therefore need to estimate $(a)$ the frequencies of pairs and of associations of higher multiples at pachytene along the lines reported above, as well as $(b)$ the distribution of subsequent chiasmata among pairs, and among multiple associations as described in detail by John and Henderson (loc. cit.).

To turn, finally, to a related but separate issue. In view of the already high bivalent frequency of normal autotetraploids in rye it is surprising that 
a selection for improved fertility is achieved by increasing the multivalent (quadrivalent) frequency (Hazarika and Rees, 1967). The same is true for Lolium (Crowley and Rees, 1968). One would have supposed the reverse, increasing bivalents at the expense of multivalents, would be more effective and, also, more readily achieved by reinforcing the formation of exclusive pairs at pachytene. This may well be the mechanism adopted in the bivalent forming autotetraploid Lotus (Dawson, 1941) as, undoubtedly, it is in the diploidised " allotetraploid" wheat (Riley, 1960; Feldman, 1966).

\section{Summary}

1. At meiosis in most pollen mother cells of autotetraploid rye lines the four chromosomes of one of the seven homologous sets associate exclusively in "pairs" at pachytene.

2. The tendency among chromosome multiples to form exclusive pachytene pairs is confirmed by observations in trisomic rye and in interchange heterozygotes.

3. The restriction of some associations to pairs at pachytene contributes to the paucity of multivalents characteristic of rye and many other autotetraploids.

4. A method is described for estimating the frequency of chromosome pairs at pachytene. This information, coupled with information on the distribution of chiasmata, permits of detailed predictions of metaphase configurations.

\section{REFERENCES}

CALLAN, H. G. 1949. Chiasma interference in diploid, tetraploid and interchange spermatocytes of the earwig Forficula auricularia. F. Genet., 49, 209-213.

CROWLEY, J. G., AND REES, H. 1968. Fertility and selection in tetraploid Lolium. Chromosoma (Berl.), 24, 300-308.

DARLINGton, c. D. 1937. Recent Advances in Cytology, pp. $671+\mathrm{xvi}$, Churchill.

DAwson, C. D. R. 1941. Tetrasomic inheritance in Lotus corniculatus L. F. Gen., 42, 49-72.

DURRANT, A. 1960. Expected frequencies of chromosome associations in tetraploids with random chiasma formation. Genetics, 45, 779-783.

FELDMAN, M. 1966. The effect of chromosomes $5 B, 5 D$ and $5 A$ on chromosomal pairing in Triticium aestivum. P.N.A.S., 55, 1447-1453.

HAZARIKA, M. H., AND REES, H. 1967. Genotypic control of chromosome behaviour in rye. $\mathrm{X}$. Chromosome pairing and fertility in autotetraploids. Heredity, 22, 317-332.

JOHN, B., AND HENDERSON, s. A. 1962. Asynapsis and polyploidy in Shistocerca paranensis. Chromosoma (Berl.), 13, 111-147.

MORRISON, J. W., AND RAJHATHY, T. 1960. Chromosome behaviour in autotetraploid cereals and grasses. Chromosoma (Berl.), 11, 297-309.

REES, H. 1955. Genotypic control of chromosome behaviour in rye. I. Inbred lines. Heredity, 9, 93.

REES, H. 1961. The consequences of interchange. Evolution, 15, 145-152.

RILEY, R. 1960. The diploidisation of polyploid wheat. Heredity, 15, 407.

THOMPSON, J. в. 1956. Genotypic control of chromosome behaviour in rye. II. Disjunction at meiosis in interchange heterozygotes. Heredity, 10, 99-108. 